\title{
Elevated Serum Vascular Cell Adhesion Molecule-1 Is Associated with Septic Encephalopathy in Adult Community-Onset Severe Sepsis Patients
}

\author{
Chih-Min Su, ${ }^{1,2}$ Hsien-Hung Cheng, ${ }^{2}$ Tsung-Cheng Tsai, ${ }^{2}$ Sheng-Yuan Hsiao, ${ }^{2}$ \\ Nai-Wen Tsai, ${ }^{3}$ Wen-Neng Chang, ${ }^{3}$ Wei-Che Lin, ${ }^{4}$ Ben-Chung Cheng, ${ }^{1,5}$ Yu-Jih Su, ${ }^{1,5}$ \\ Ya-Ting Chang, ${ }^{1,3}$ Yi-Fang Chiang, ${ }^{3}$ Chia-Te Kung, ${ }^{2}$ and Cheng-Hsien Lu ${ }^{1,3}$ \\ ${ }^{1}$ Department of Biological Science, National Sun Yat-Sen University, Kaohsiung 80424, Taiwan \\ ${ }^{2}$ Department of Emergency Medicine, Kaohsiung Chang Gung Memorial Hospital, Chang Gung University College of Medicine, \\ No. 123, Ta Pei Road, Niao Sung Hsiang, Kaohsiung 833, Taiwan \\ ${ }^{3}$ Department of Neurology, Kaohsiung Chang Gung Memorial Hospital, Chang Gung University College of Medicine, \\ No. 123 Ta Pei Road, Niao Sung Hsiang, Kaohsiung 833, Taiwan \\ ${ }^{4}$ Department of Radiology, Kaohsiung Chang Gung Memorial Hospital, Chang Gung University College of Medicine, \\ No. 123, Ta Pei Road, Niao Sung Hsiang, Kaohsiung 833, Taiwan \\ ${ }^{5}$ Department of Internal Medicine, Kaohsiung Chang Gung Memorial Hospital, Chang Gung University College of Medicine, \\ No. 123, Ta Pei Road, Niao Sung Hsiang, Kaohsiung 833, Taiwan
}

Correspondence should be addressed to Cheng-Hsien Lu; chlu99@ms44.url.com.tw

Received 26 February 2014; Revised 11 April 2014; Accepted 11 April 2014; Published 6 May 2014

Academic Editor: Hung-Chen Wang

Copyright (c) 2014 Chih-Min Su et al. This is an open access article distributed under the Creative Commons Attribution License, which permits unrestricted use, distribution, and reproduction in any medium, provided the original work is properly cited.

\begin{abstract}
Background and Aim. Septic encephalopathy (SE) is a common complication of severe sepsis. Increased concentrations of circulating soluble adhesion molecules are reported in septic patients. This study aimed to determine whether serum adhesion molecules are associated with SE. Methods. Seventy nontraumatic, nonsurgical adult patients with severe sepsis admitted through ER were evaluated. Serum adhesion molecules were assessed for their relationship with SE, and compared with other clinical predictors and biomarkers. Results. Twenty-three (32.8\%) patients had SE. SE group had higher in-hospital mortality ( $40 \%$ versus $11 \%, P=0.009)$ and their sVCAM-1, sICAM-1, and lactate levels on admission were also higher than non-SE group. By stepwise logistic regression model, sVCAM-1, age, and maximum 24-hours SOFA score were independently associated with septic encephalopathy. The AUC analysis of ROC curve of different biomarkers showed that sVCAM-1 is better to predict SE. The sVCAM-1 levels in the SE group were significantly higher than those of the non-SE group at three time periods (Days 1, 4, and 7). Conclusions. Septic encephalopathy implies higher mortality in nontraumatic, nonsurgical patients with severe sepsis. VCAM-1 level on presentation is a more powerful predictor of SE in these patients than lactate concentration and other adhesion molecules on admission.
\end{abstract}

\section{Introduction}

Septic encephalopathy (SE) is a common complication of severe sepsis and septic shock. An estimated $9-71 \%$ of patients with sepsis exhibit symptoms of encephalopathy [13], including consciousness disturbance, impaired cognitive function, personality changes, and lack of concentration or somnolence $[4,5]$. Several mechanisms have been proposed, including oxidative stress [6], increased cytokine and proinflammatory factor [7], mitochondrial dysfunction, apoptosis [8], decreased cerebral blood flow [9], endothelium activation [10], and blood-brain barrier breakdown [11], as well as any combination of the abovementioned etiologies. By itself, SE is pivotal in determining sepsis mortality [12] and makes clinical evaluation more complex since patients cannot express themselves well. Although SE has been described as a reversible syndrome, studies indicate longlasting cognitive and depressive disturbances in patients 
after the sepsis resolves [13]. Early accurate detection of septic encephalopathy not only makes clinical physician more alert about the sepsis status and aggressive treatment but also decreases unnecessary examination and movement of patient.

To date, useful biomarkers in predicting SE are still limited. Several studies used S-100B protein, a marker of astrocytes activation and injury, as a marker for brain injury in SE $[14,15]$. However, its results were not promising. Five studies demonstrated that elevated S-100B protein correlated with the development of SE, but two other studies found no correlation and all their sample sizes were small $[16,17]$. On the other hand, studies using animal models showed that some proinflammatory cytokines or cell adhesion molecules could be potential biomarkers that induce SE, but their effects on human beings are still not demonstrated [18-20].

In human studies, increased concentrations of circulating soluble adhesion molecules have been reported in patients with systemic inflammatory response syndrome, septic shock, and cardiovascular diseases [21, 22]. Increased concentrations of adhesion molecules have also been associated with multiple organ dysfunction, disease severity, or death [23]. Since sVCAM-1 and sICAM-1 have been involved in leukocyte-endothelium cell crosstalk at the blood-brain barrier [24], we wanted to know their relationship with SE. This prospective study aimed to determine the roles of serum adhesion molecules and conventional biomarkers in predicting SE among adult severe sepsis patients.

\section{Patients and Methods}

2.1. Study Population. This is a secondary analysis of prospective collected data on the time course of levels of adhesion molecules in severe sepsis and septic shock patients and the association of these biomarkers with SE. Over an 18-month period (January 2011 to June 2012), patients aged $\geq 20$ years who were admitted through emergency room (ER) of Kaohsiung Chang Gung Memorial Hospital (CGMH), a 2482-bed acute-care teaching hospital in southern Taiwan providing both primary and tertiary referral care, were screened every weekday for severe sepsis and septic shock according to specific criteria and were enrolled in the study within 24 hours after identification. The hospital's Institutional Review Committee on Human Research approved the study, and all of the patients provided informed consent.

Severe sepsis on ER admission was defined according to the American College of Chest Physicians/Society of Critical Care Medicine criteria, which included the following: (a) suspicion or confirmed infection; (b) two or more manifestations of systemic inflammatory response syndrome; and (c) at least one sepsis-induced acute organ dysfunction or signs of hypoperfusion. All of the patients who met these three criteria were eligible to enroll in the study. Septic shock was defined as severe sepsis associated with hypotension not controlled by vascular expansion but requiring vasopressive agents to maintain SBP > $90 \mathrm{mmHg}$ [25].

Patients were excluded if they had one of the following: (1) traumatic etiology; (2) previous surgical treatment;
(3) underlying hematologic diseases or those under chemotherapy; (4) pregnancy; (5) central nervous systems disorders with various levels of conscious disturbance before arriving at the ER; and (6) history of exposure to drug, toxic substances, alcohol, industrial agents, heavy metals, or any substance known to cause consciousness change. We excluded these patients to eliminate the influence of inflammation reaction other than sepsis on the biomarkers and reduced the potential factors that would affect the diagnosis of SE.

2.2. Clinical Assessment and Treatment. The patient information collected was demographic data, Acute Physiology and Chronic Health Evaluation (APACHE) II score, Sequential Organ Failure Assessment (SOFA) score, and Charlson Comorbidity Index (CCI) score, which were calculated during the first 24 hours of admission to assess the severity of organ dysfunction. Basic laboratory tests, lactate concentration, B-type natriuretic peptide, and inflammatory markers, including plasma C-reactive protein (CRP) and procalcitonin, were taken on ER admission. The course of various organ dysfunctions and supportive treatments like vasoactive drugs, ventilator, and steroid therapies were also recorded.

2.3. Definition. Comorbidity was defined as preexisting disorder before severe sepsis event. Stroke history in this study only referred to the patients who still could maintain clear conscious level after ischemic or hemorrhagic stroke event. Charlson comorbidity scoring system was used to assess the severity of comorbidity [26]. The source of infection was classified as one of the following: lower respiratory tract, urinary tract, skin and musculoskeletal soft tissue, central nervous system, or intra-abdomen infection which included liver abscess, spontaneous bacterial peritonitis, biliary tract, peritonitis, and primary bacteraemia with unknown focus. For the grading of disease severity, we use both $24 \mathrm{~h} \mathrm{APACHE}$ II and $24 \mathrm{~h}$ SOFA score which were calculated according to their laboratory data and clinical parameters [27, 28]. And mortality in this study means in-hospital mortality. Ventilator treatment within 24 hours means that the patient needed ventilator support to help him or her maintain adequate oxygenation and the event happened within 24 hours of ER admission.

2.4. Septic Encephalopathy. The patient's conscious level was recorded by the Glasgow Coma Scale (GCS) and mental status at least twice daily. Symptoms of SE included somnolence, stupor, coma, confusion, disorientation, agitation, irritability, and decreased level of GCS. Encephalopathy was confirmed if the patient had two or more of the aforementioned symptoms for more than 72 hours, regained consciousness after treatment, or deteriorated and died. Continuous sedative medication was never used, even in mechanically ventilated patients, although short-duration sedatives were used if patients did not cooperate for the treatment and examination. Patients with obvious etiologies of consciousness change other than SE during treatment were excluded. These include severe hypoglycemia event, intracranial hemorrhage, status 
epilepsy, acute ischemic stroke, hyponatremia, and cardiopulmonary resuscitation related hypoxic encephalopathy.

2.5. Assessment of Infectious Biomarkers. All tests were conducted by the quality-controlled central laboratory of CGMH. Concentrations of CRP were determined by enzyme immunoassay (EMIT; Merck Diagnostica; Zurich, Switzerland), while PCT was measured using enzyme-linked fluorescent assay (VIDAS; BioMerieux; Ponte a Ema, Italy). Serum lactate levels were measured using a serum-based assay catalyzed by lactate oxidase (UniCel Integrated System; Beckman Coulter INS; Boulevard, Brea, CA).

2.6. Blood Sampling and Assessment of Serum Adhesion Molecules. Blood samples of serum adhesion molecules were collected on the first day of enrollment (Day 1). Additional samples were obtained on Days 4 and 7. Blood samples were collected by venipuncture into Vacutainer SST tubes. Blood was allowed to clot in room temperature for a minimum of 30 minutes, and the clot was removed by immediate centrifugation at 3,000 rpm for $10 \mathrm{~min}$ at $4^{\circ} \mathrm{C}$. All serum samples were collected after centrifugation, isolated, and stored at $-80^{\circ} \mathrm{C}$ in multiple aliquots. Serum sICAM-1, sVCAM-1, sE-selectin, sL-selectin, and sP-selectin levels were determined by commercially available ELISA (R\&D Systems, Minneapolis, MN, USA). In the assay, standards, controls, and unknown samples were incubated in microtitration wells that were coated with marked antibodies (i.e., anti-ICAM1, VCAM-1, P-selectin, E-selectin, and L-selectin). After incubation and washing, the wells were treated with another anti-Ag detection antibody labeled with enzyme horseradish peroxidase (HRP).

After second incubation and washing, the wells were incubated with the substrate tetramethylbenzidine (TMB). An acidic stopping solution was then added, and the enzymatic turnover rate of the substrate was determined by dual wavelength absorbance measured at 450 and $620 \mathrm{~nm}$. Absorbance was directly proportional to the concentration of antigens present. A set of antigen standards was used to plot a standard curve of absorbance versus antigen concentration, from which antigen concentrations in the unknowns were calculated.

Samples for serum adhesion molecules were collected and measured immediately. It takes 5 hours to measure serum levels. Other serum samples were collected after centrifugation, isolated, and stored at $-80^{\circ} \mathrm{C}$ in multiple aliquots in accordance with our study design [29].

2.7. Statistical Analysis. Data were presented as mean \pm SD or $n$ (\%) accordingly. Comparisons between septic encephalopathy and no encephalopathy groups were made by MannWhitney $U$ test, while proportions among groups were compared by using $\chi^{2}$ test or Fisher's exact test. Repeated measures of ANOVA were used to compare serum adhesion molecules at three different time points after severe sepsis. Analysis of covariance (ANCOVA) was used to compare groups after controlling for potential confounding variables. Spearman correlation analysis was used to test the correlation of serum adhesion molecule levels and traditional infection makers with the severity of sepsis, including maximum $24 \mathrm{~h}$ SOFA score and maximum $24 \mathrm{~h}$ APACHE II score.

Stepwise logistic regression was used to evaluate the relationship between significant variables and septic encephalopathy, with adjustments for other potential confounding factors. Only variables strongly associated with septic encephalopathy $(P<0.05)$ were included in the final model. Receiver operating characteristic (ROC) curves were generated to determine a cut-off level for significant variables for SE. Areas under the ROC curves (AUCs) were calculated for each parameter and compared. All statistical analyses were conducted using the SAS software package, version 9.1 (2002, SAS Statistical Institute, Cary, NC).

\section{Results}

3.1. Baseline Characteristics of the Study Patients. Seventy-five adult severe sepsis and septic shock patients were enrolled, but five patients were excluded after finding the etiology of consciousness change. Two of them had severe hypoglycemia, and the other 3 patients had severe hyponatremia, basilar artery occlusion as revealed by brain magnetic resonance imaging, and status epilepsy by electroencephalography, respectively. In the 70 patients, there were 22 females and 48 males, with an average age of 64.3 years. During admission, 23 patients experienced SE and 47 patients did not. Among the 23 SE events, 14 (61\%) occurred at the time of enrollment, $6(26 \%)$ were within $24 \mathrm{~h}$ after enrollment, and 3 (13\%) were after $24 \mathrm{~h}$ but within 3 days of enrollment. The 14 patients who received brain computed tomography (CT) examination all had nonspecific findings. Four patients died after SE, but another five patients had returned to clear consciousness before occurrence of death.

Baseline characteristics, including comorbidities, clinical presentations, hospital mortality, and disease severity index including shock within $24 \mathrm{~h}$, mechanical ventilation treatment within $24 \mathrm{~h}$, and maximum $24 \mathrm{~h}$ APACHE II and $24 \mathrm{~h}$ SOFA scores between the SE and non-SE groups, were listed in Table 1. In this study, the patient with a stroke history was more likely to have $\mathrm{SE}$ (odds ratio (OR) 5.6, $P=0.03$ ). In the disease severity index, the SE group had higher APACHE II and SOFA scores $(21.3 \pm 5.5$ versus $17.5 \pm 5.7, P=0.01 ; 8.2 \pm 2.4$ versus $5.4 \pm 3.1, P<0.001$, resp.), which meant more organ dysfunction. In-hospital mortality and ventilator treatment within 24 hours were also both higher in SE patients $(40 \%$ versus $11 \%, P=0.009 ; 57 \%$ versus $24 \%, P=0.008$, resp.).

The sources of infection and laboratory data of the two groups were listed in Tables 2 and 3, respectively. There was no significant difference between infection source and culture result. Serum lactate $(50.5 \pm 37.6$ versus $32.6 \pm 20.3, P=0.05)$, sICAM-1 (1028.2 \pm 525.2 versus $764.8 \pm 504.9, P=0.03)$, and sVCAM-1 $1_{\text {Dayl }}(3048.1 \pm 1261.1$ versus $1969.0 \pm 1129.5, P=$ 0.001 ) were the only three markers with significant difference between the SE and non-SE groups.

3.2. Effect of Infection Markers and Serum Adhesion Molecules on Sepsis Severity. Based on the statistical results (Spearman 
TABLE 1: Baseline characteristics of septic encephalopathy (SE) and non-SE groups in severe sepsis patients.

\begin{tabular}{|c|c|c|c|}
\hline & $\begin{array}{l}\text { SE group } \\
n=23\end{array}$ & $\begin{array}{c}\text { Non-SE group } \\
n=47\end{array}$ & $P$ value \\
\hline Age $(y)($ mean \pm SD $)$ & $68.0 \pm 11.2$ & $62.6 \pm 13.6$ & 0.15 \\
\hline Male/female & $17 / 6$ & $31 / 16$ & 0.59 \\
\hline \multicolumn{4}{|l|}{ Underlying diseases $[n(\%)]$} \\
\hline Diabetes mellitus & $10(44)$ & $15(32)$ & 0.43 \\
\hline Hypertension & $8(35)$ & $22(47)$ & 0.44 \\
\hline Liver diseases/alcoholism & $6(26)$ & $6(13)$ & 0.19 \\
\hline Chronic lung disease & $3(13)$ & $11(23)$ & 0.36 \\
\hline Stroke $\mathrm{a}^{\mathrm{a}}$ & $7(30)$ & $4(9)$ & $0.03^{*}$ \\
\hline Coronary artery disease & $0(0)$ & $6(13)$ & 0.17 \\
\hline Cancer & $6(21)$ & $10(21)$ & 0.76 \\
\hline Chronic renal disease & $2(9)$ & $1(2)$ & 0.25 \\
\hline \multicolumn{4}{|l|}{ Clinical presentations $($ mean $\pm \mathrm{SD})$} \\
\hline Systolic BP & $108.0 \pm 39.0$ & $108.0 \pm 47.0$ & 0.91 \\
\hline Diastolic BP & $72.1 \pm 24.2$ & $64.2 \pm 22.8$ & 0.20 \\
\hline Pulse rate & $103 \pm 26$ & $112 \pm 25$ & 0.26 \\
\hline Respiratory rate & $20 \pm 3$ & $21 \pm 4$ & 0.25 \\
\hline Shock within 24 hours $[n(\%)]$ & $15(65)$ & $34(74)$ & 0.58 \\
\hline Ventilator treatment within 24 hours $[n(\%)]$ & $13(57)$ & $11(24)$ & $0.008^{*}$ \\
\hline \multicolumn{4}{|l|}{ Disease severity index $($ mean $\pm \mathrm{SD})$} \\
\hline Maximum 24 h APACHE II score & $21.3 \pm 5.5$ & $17.5 \pm 5.7$ & $0.01^{*}$ \\
\hline CCI score & $3.6 \pm 2.6$ & $3.2 \pm 3.0$ & 0.44 \\
\hline Maximum 24 h SOFA score & $8.2 \pm 2.4$ & $5.4 \pm 3.1$ & $<0.001^{*}$ \\
\hline Steroid treatment $[n(\%)]$ & $11(48)$ & $21(45)$ & 1.00 \\
\hline In-hospital mortality $[n(\%)]$ & $9(40)$ & $5(11)$ & $0.009^{*}$ \\
\hline
\end{tabular}

SD: standard deviation; BP: blood pressure; APACHE: Acute Physiology and Chronic Health Evaluation; CCI: Charlson Comorbidity Index; SOFA: Sequential Organ Failure Assessment.

${ }^{a}$ Stroke only referred to the patients who still could maintain clear conscious level after ischemic or hemorrhagic stroke event.

${ }^{*} P<0.05$.

correlation coefficient, $P$ value $)$, $\operatorname{sICAM-1}$ Dayl level $(\rho=0.36$, $P=0.003)$, sVCAM-1 $1_{\text {Dayl }}$ level $(\rho=0.404, P=0.001)$, sE-selectin $_{\text {Dayl }}(\rho=0.364, P=0.002)$, procalcitonin $(\rho=$ $0.347, P=0.004)$, and lactate $(\rho=0.379, P=0.001)$ had correlation with maximum $24 \mathrm{~h}$ SOFA score. sE-selectin ${ }_{\text {Dayl }}$ $(\rho=0.284, P=0.02), \operatorname{sVCAM} 1_{\text {Dayl }}(\rho=0.287, P=$ $0.018)$, and lactate $(\rho=0.441, P<0.001)$ had correlation with maximum $24 \mathrm{~h}$ APACHE II score. Traditional infection markers, CRP, were neither related to SOFA nor to APACHE II score.

3.3. Prediction of Septic Encephalopathy. Serum sVCAM-1 $1_{\text {Dayl }}$ level, sICAM-1 ${ }_{\text {Dayl }}$ level, serum lactate level, $24 \mathrm{~h}$ SOFA score, $24 \mathrm{~h}$ APACHE II score, stroke history, and ventilator treatment within 24 hours were significantly higher in SE patients and could be used as clinical predictors. However, after using both forward and backward stepwise logistic regression model with all the predictors plus age and sex, only sVCAM-1 $1_{\text {Day } 1}$ level $(P=0.009,0.02)$, age $(P=0.002$, $0.011)$, and SOFA score $(P=0.007,0.002)$ were independently associated with SE.
The effectiveness of infection markers in predicting SE in the ER setting was evaluated by assessing the area under curve (AUC) of each biomarker's ROC curves. The AUCs for each marker were calculated (Table 4; Figure 1). The AUC for CRP, procalcitonin, lactate, E-selectin ${ }_{\text {Dayl }}$, sICAM-1 $1_{\text {Day1 }}$, and sVCAM- $1_{\text {Dayl }}$ levels was $0.561(P=0.423), 0.616(P=0.130)$, $0.647(P=0.052), 0.593(P=0.219), 0.664(P=0.031)$, and $0.760(P=0.001)$, respectively. $s$ VCAM-1 $1_{\text {Dayl }}$ level had the highest AUC, reflecting good discrimination. Our suggestion of $s$ VCAM-1 ${ }_{\text {Dayl }}$ cut-off value for predicting SE was $1900 \mathrm{ng} / \mathrm{mL}$ for the better sensitivity (sensitivity of $81.8 \%$, specificity of $61.9 \%$, positive predictive value of $51.2 \%$, and negative predictive value of $87.4 \%$ ).

3.4. Time Course of Serum Vascular Cell Adhesion Molecule Levels. Serum sVCAM-1 levels obtained at different days (Figure 2) revealed that sVCAM-1 level gradually decreased in both groups. In all three different testing points, the SE group had significantly higher sVCAM-1 level than the non-SE group using Mann-Whitney $U$ test $(3048.1 \pm 1261.1$ versus $1969.0 \pm 1129.5, P=0.001 ; 3011.5 \pm 1158.1$ versus 
TABLE 2: Sources of infection in septic encephalopathy (SE) and non-SE groups.

\begin{tabular}{|c|c|c|c|}
\hline Sources of infection & $\begin{array}{c}\text { SE group } \\
n=23\end{array}$ & $\begin{array}{c}\text { Non-SE group } \\
n=47\end{array}$ & $P$ value \\
\hline Respiratory tract infection & $8(36.4)$ & $19(40.4)$ & 0.955 \\
\hline Urinary tract infection & $4(18.2)$ & $9(19.1)$ & \\
\hline Intra-abdominal infection & $4(18.2)$ & $10(21.3)$ & \\
\hline Soft tissue infection & $5(22.7)$ & $7(14.9)$ & \\
\hline Unknown origin & $1(4.5)$ & $2(4.3)$ & \\
\hline Concurrent bacteremia episode & $13(56.5)$ & $21(44.7)$ & 0.192 \\
\hline Causative pathogens & & & 0.56 \\
\hline \multicolumn{4}{|l|}{ Gram-negative } \\
\hline Escherichia coli & $5(21.7)$ & $6(12.8)$ & \\
\hline Klebsiellapneumoniae & $2(8.7)$ & $8(17.0)$ & \\
\hline Proteus mirabilis & $1(4.3)$ & $1(2.1)$ & \\
\hline Burkholderiapseudomallei & $1(4.3)$ & $0(0)$ & \\
\hline Salmonella enteritidis & $1(4.3)$ & $0(0)$ & \\
\hline \multicolumn{4}{|l|}{ Gram-positive } \\
\hline Streptococcus pneumoniae & $0(0)$ & $2(4.3)$ & \\
\hline$\beta$-Hemolytic Streptococcus group A & $1(4.3)$ & $2(4.3)$ & \\
\hline Staphylococcus aureus & $2(8.7)$ & $2(4.3)$ & \\
\hline
\end{tabular}

TABLE 3: Laboratory data of the septic encephalopathy (SE) and non-SE groups in severe sepsis patients.

\begin{tabular}{|c|c|c|c|}
\hline & $\begin{array}{c}\text { SE group } \\
n=23\end{array}$ & $\begin{array}{c}\text { Non-SE group } \\
n=47\end{array}$ & $P$ value \\
\hline \multicolumn{4}{|l|}{$\mathrm{WBC} / \mathrm{DC}($ mean $\pm \mathrm{SD})$} \\
\hline $\mathrm{WBC}\left(\times 10^{9} / \mathrm{L}\right)$ & $15.0 \pm 10.0$ & $17.7 \pm 12.0$ & 0.13 \\
\hline Segment (\%) & $76.7 \pm 20.3$ & $81.8 \pm 17.3$ & 0.38 \\
\hline Band $(\%)$ & $8.5 \pm 12.7$ & $3.2 \pm 4.8$ & 0.06 \\
\hline Lymphocyte (\%) & $7.7 \pm 7.9$ & $10.4 \pm 17.4$ & 0.96 \\
\hline \multicolumn{4}{|l|}{ Biochemistry (mean \pm SD) } \\
\hline Glucose $(\mathrm{mg} / \mathrm{dL})$ & $210 \pm 162$ & $171 \pm 94$ & 0.91 \\
\hline Creatinine (mg/dL) & $2.9 \pm 1.9$ & $2.3 \pm 2.5$ & 0.06 \\
\hline Lactate $(\mathrm{mg} / \mathrm{dL})$ & $50.5 \pm 37.6$ & $32.6 \pm 20.3$ & $0.05^{*}$ \\
\hline Total bilirubin (mg/dL) & $3.3 \pm 3.0$ & $2.2 \pm 2.7$ & 0.09 \\
\hline $\mathrm{BNP}(\mathrm{pg} / \mathrm{mL})$ & $991 \pm 1127$ & $742 \pm 1024$ & 0.21 \\
\hline \multicolumn{4}{|c|}{ Inflammatory markers $($ mean $\pm S D)$} \\
\hline $\mathrm{CRP}(\mathrm{mg} / \mathrm{L})$ & $231 \pm 112$ & $206 \pm 137$ & 0.40 \\
\hline Procalcitonin (ng/mL) & $32.5 \pm 33.4$ & $35.6 \pm 59.4$ & 0.12 \\
\hline sICAM-1 Dayl $(\mathrm{ng} / \mathrm{mL})$ & $1028.2 \pm 525.2$ & $764.8 \pm 504.9$ & $0.03^{*}$ \\
\hline $\mathrm{sVCAM} \mathrm{1}_{\text {Dayl }}(\mathrm{ng} / \mathrm{mL})$ & $3048.1 \pm 1261.1$ & $1969.0 \pm 1129.5$ & $0.001^{*}$ \\
\hline sP-selectin $_{\text {Dayl }}(\mathrm{ng} / \mathrm{mL})$ & $98.8 \pm 42.5$ & $98.0 \pm 23.0$ & 0.77 \\
\hline sL-selectin $_{\text {Dayl }}(\mathrm{ng} / \mathrm{mL})$ & $1208.2 \pm 777.5$ & $933.9 \pm 311.9$ & 0.10 \\
\hline $\mathrm{sE}$ selectin $_{\text {Dayl }}(\mathrm{ng} / \mathrm{mL})$ & $214.2 \pm 111.9$ & $117.8 \pm 127.6$ & 0.22 \\
\hline
\end{tabular}

WBC: white blood cells; DC: differential count; CRP: C-reactive protein; BNP: B-type natriuretic peptide; sE-selectin: soluble E-selectin; sICAM-1: soluble intercellular adhesion molecule-1; sL-selectin: soluble L-selectin; sP-selectin: soluble P-selectin; sVCAM-1: soluble vascular cell adhesion molecule-1. ${ }^{*} P<0.05$. 
TABLE 4: AUC for biomarkers in diagnosing septic encephalopathy by ROC curve analysis.

\begin{tabular}{llcr}
\hline & AUC & $95 \%$ confidence interval & $P$ value \\
\hline CRP & 0.561 & $0.416-0.705$ & 0.423 \\
Procalcitonin & 0.616 & $0.479-0.752$ & 0.130 \\
Lactate & 0.647 & $0.494-0.800$ & 0.052 \\
sVCAM-1 & 0.760 & $0.638-0.881$ & $0.001^{*}$ \\
sICAM-1 & 0.664 & $0.532-0.795$ & $0.031^{*}$ \\
sE-selectin & 0.593 & $0.452-0.734$ & 0.219 \\
\hline
\end{tabular}

AUC: area under the curve; CRP: C-reactive protein; sVCAM-1: soluble vascular cell adhesion molecule-1; sE-selectin: soluble E-selectin; sICAM-1: soluble intercellular adhesion molecule-1; ROC: receiver operating characteristic.

${ }^{*} P<0.05$.

$1532.6 \pm 1005.2, P<0.001 ; 2376.4 \pm 1017.9$ versus $1597.6 \pm$ 1048.0, $P=0.027$, resp.). By repeated measures of ANOVA, serum VCAM-1 levels between the two groups at three different time points, Days 1, 4, and 7, were significantly different $(P=0.011)$.

\section{Discussion}

The present study examines the association between SE and circulatory cell adhesion molecules and produces the following major findings. First, the level of serum sVCAM1, sICAM-1, and lactate on presentation, $24 \mathrm{~h}$ SOFA score, $24 \mathrm{~h}$ APACHE II score, underlying diseases of stroke, and ventilator treatment within 24 hours were significantly higher in the SE group of severe septic patients than in the nonSE group. Second, VCAM-1 level on presentation is a more powerful predictor of SE in severe sepsis patients than lactate concentration and other adhesion molecules on admission by stepwise logistic regression and AUC analysis. Lastly, sVCAM-1 levels in the SE group were significantly higher than those of the non-SE group at three time periods (Days 1, 4 , and 7).

In this study, SE is closely related to the severity and mortality of severe sepsis and septic shock. The $24 \mathrm{~h}$ APACHE II score, SOFA score, serum lactate level, ventilator treatment within 24 hours, and mortality rate in SE of severe septic patients are significantly higher than those of nonSE patients. The longer duration of SE also implies higher mortality since four patients died within three more days of SE. Our result is compatible with other studies [30]. In another study, they also found that mortality related to SE increased from $16 \%$ to $63 \%$ when the GCS decreased from 15 to less than 8 [12]. The increased mortality in SE group could partially explained by increased severity of sepsis. But further evaluation of the direct effect of brain injury to the systemic response may need to clarify it.

Patients with SE had also higher incidence of early respiratory failure. In clinical practice, patients with conscious disturbance are at risk of respiratory failure since they have less airway protection and respiratory drives. Certain periods of hypoxemia before respiratory failure may also cause more brain injury. Tissue hypoperfusion can lead to elevated serum lactate level and increased acidosis, which can explain part of the mechanisms of SE by their correlation.
Cell adhesion molecules regulate endothelial function by activating leukocyte recruitment and tissue inflammation. Endothelium dysfunction may be central to the development of sepsis-induced multiple organ failure [31]. Soluble form of VCAM-1, ICAM-1, and E-selectin are present in plasma and reflect cellular inflammatory status and correlate with endothelial dysfunction [32]. In the present study, all three kinds of cell adhesion molecules and serum lactate correlate with SOFA score. They can represent the degree of organ damage in severe sepsis and septic shock patients.

Brain has a unique blood-brain barrier that regulates the brain capillary blood flow and thus precisely maintains the brain internal microenvironment [33]. But endothelial activation may result in the breakdown of the blood-brain barrier [34]. Increased permeability of the blood-brain barrier has been demonstrated in experimental models of sepsis [35]. The brain MRI of SE patients also reveals vasogenic edema of the brain parenchyma $[36,37]$. Hofer's study using septic animal models reveals early changes in the integrity of the blood-brain barrier in the central nervous system. Increased cerebral ICAM-1 expression may be an early factor involved in these pathogenic events [20]. In Hamed's study, children with sepsis-induced encephalopathy have elevated serum and cerebrospinal fluid levels of sICAM-1, NO, and S100B compared to those with sepsis only [38]. Both studies used only sICAM-1 as the biomarkers.

In our study, both sICAM-1 and sVCAM-1 had correlated with SE. Serum sVCAM-1 and sICAM-1 are also correlated with each other in this study. Previous study has revealed that many signaling events attributed to ICAM-1 engagement appear to be similar to VCAM-1 [39]. It is difficult to disentangle the two CAMs effects. In cardiovascular disease study, sICAM appears to be a general marker of proinflammation and may be used as a risk factor. But sVCAM-1, which is not expressed in baseline conditions, is rapidly induced by injury and can emerge as a strong risk predictor of existing disease [40]. Compared to sICAM-1 and E-selectin, it is sVCAM-1 that has the strongest correlation with cardiovascular-related future death and cardiovascular events [41]. Just like our study, sVCAM-1 is also a better predictor than other adhesion molecules in SE patients.

4.1. Limitations. Although this study demonstrates that serum VCAM-1 level on presentation is a more powerful 

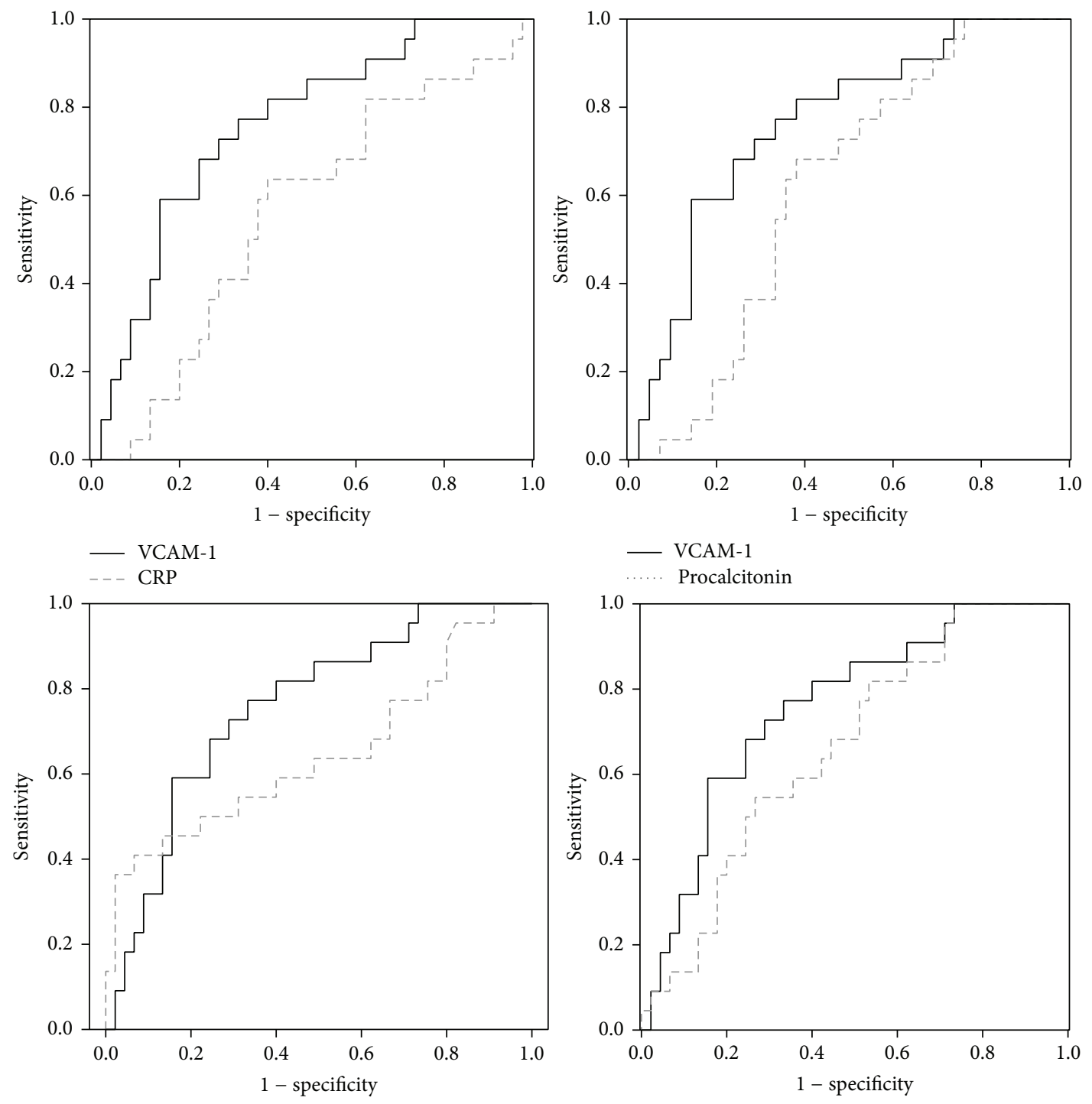

- VCAM-1

- VCAM-1

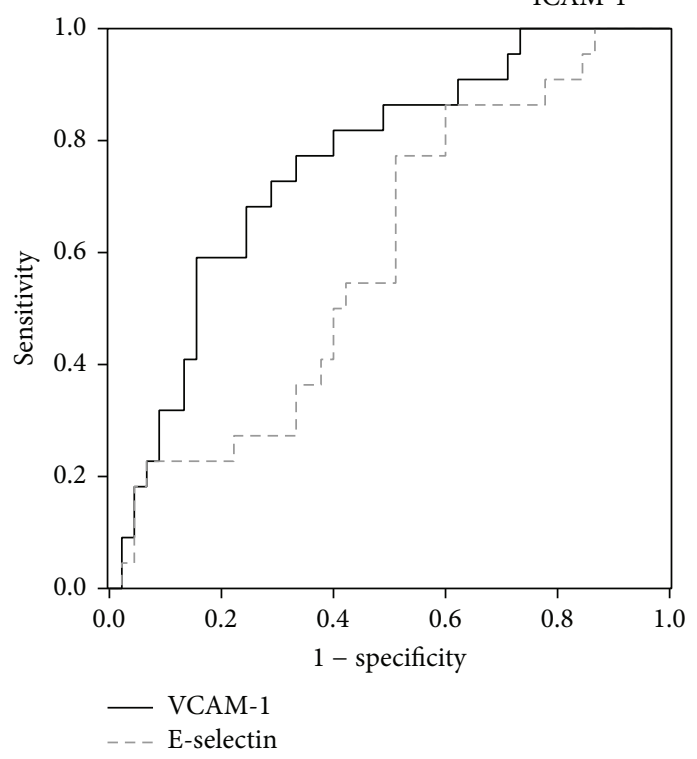

FIGURE 1: The comparison ROC curve of various biomarkers with SVCAM-1 for predicting septic encephalopathy. 


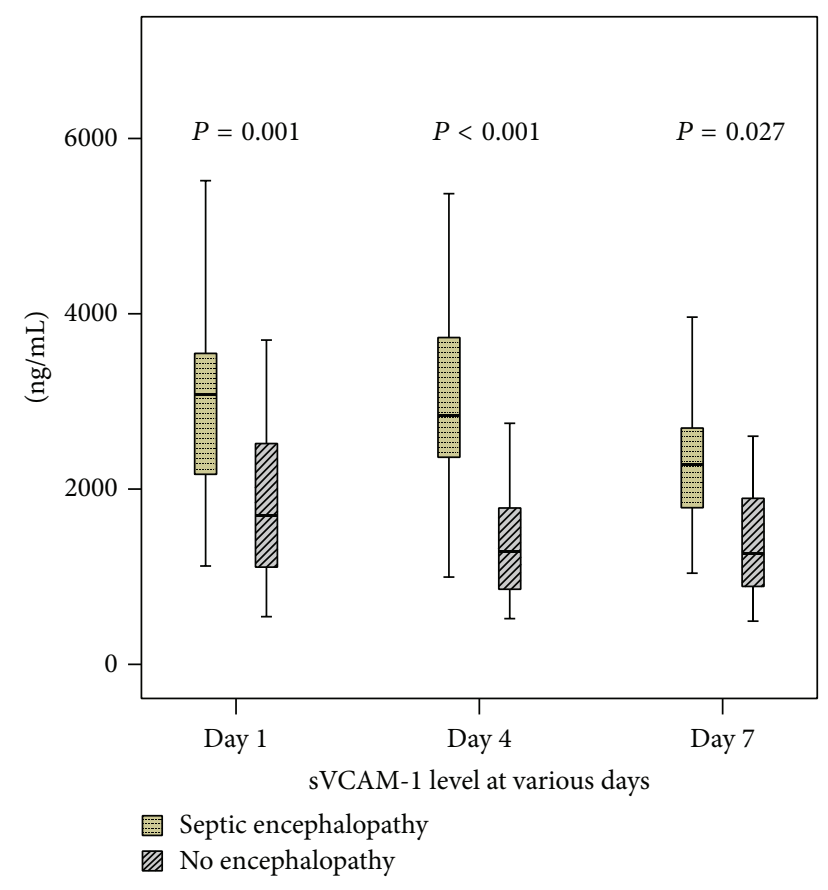

Figure 2: Comparison of serum VCAM-1 level between septic encephalopathy (SE) and non-SE groups in various days.

predictor of SE in severe sepsis patients than lactate concentration and other adhesion molecules on admission, this study has several limitations. First, the occurrence of SE can be at the time of presentation or after the stay at the ER and both the duration and severity of SE were different in each patient. Its relationship with levels of serum cell adhesion molecules cannot be exactly evaluated in every SE patient. Second, although only nontraumatic, nonsurgical septic patients are enrolled and patients with underlying brain pathologies, cognitive decline, and continuous sedative medication use have been excluded, not all of the severe sepsis patients received both neuroimaging studies and electroencephalography studies. The findings may underestimate the "true" frequency of SE in septic patients in this study. Lastly, the choice of therapeutic strategy for sepsis (e.g., use of steroids and choice, dosage, and duration of antibiotics) may be different for each patient based on the preference of the attending physician. This may cause potential bias in patients' outcome and serum biomarkers levels.

\section{Conclusion}

In conclusion, this study demonstrates that SE implies higher mortality in severe septic patients and VCAM-1 level on presentation is a more powerful predictor of SE than lactate concentration and other adhesion molecules on admission.

\section{Ethical Approval}

The Institutional Review Committee on Human Research of Chang Gung Memorial Hospital approved the study.

\section{Conflict of Interests}

The authors declare that they have no conflict of interests.

\section{Authors' Contribution}

Chih-Min Su participated in the design of the study and drafting of the paper. Chia-Te Kung, Sheng-Yuan Hsiao, Tsung-Cheng Tsai, Hsien-Hung Cheng, Wen-Neng Chang, Ya-Ting Chang, Nai-Wen Tsai, and Hung-Chen Wang participated in the sequence alignment and clinical evaluation of patients. Wei-Che Lin interpreted the imaging studies. YiFang Chiang, Ben-Chung Cheng, and Yu-Jih Su performed the statistical analysis. Chia-Te Kung and Cheng-Hsien $\mathrm{Lu}$ conceived the study, participated in its design and coordination, and helped draft the paper. All authors read and approved the final paper. Drs. Cheng-Hsien Lu and Chia-Te Kung contributed equally to this work.

\section{Acknowledgments}

This work was supported by Grants from Chang Gung Memorial Hospital (Chang Gung Medical Research Project CMRPG891341) and NHRI-EX101-10142EI. The authors wish to thank Dr. Gene Alzona Nisperos for editing and reviewing the paper for English language considerations.

\section{References}

[1] N. W. S. Davies, M. K. Sharief, and R. S. Howard, "Infectionassociated encephalopathies-their investigation, diagnosis, and treatment," Journal of Neurology, vol. 253, no. 7, pp. 833-845, 2006.

[2] M. Ebersoldt, T. Sharshar, and D. Annane, "Sepsis-associated delirium," Intensive Care Medicine, vol. 33, no. 6, pp. 941-950, 2007.

[3] K. E. Sands, D. W. Bates, P. N. Lanken et al., "Epidemiology of sepsis syndrome in 8 academic medical centers," Journal of the American Medical Association, vol. 278, no. 3, pp. 234-240, 1997.

[4] G. Consales and A. R. de Gaudio, "Sepsis associated encephalopathy," Minerva Anestesiologica, vol. 71, no. 1-2, pp. 39-52, 2005.

[5] E. L. Streck, C. M. Comim, T. Barichello, and J. Quevedo, "The septic brain," Neurochemical Research, vol. 33, no. 11, pp. 21712177, 2008.

[6] R. M. G. Berg, K. Møller, and D. M. Bailey, "Neuro-oxidativenitrosativestressin sepsis," Journal of Cerebral Blood Flow and Metabolism, vol. 31, no. 7, pp. 1532-1544, 2011.

[7] A. Jacob, J. R. Brorson, and J. J. Alexander, "Septic encephalopathy: inflammation in man and mouse," Neurochemistry International, vol. 58, no. 4, pp. 472-476, 2011.

[8] Y.-C. Chuang, J.-L. Tsai, A. Y. W. Chang, J. Y. H. Chan, C.-W. Liou, and S. H. H. Chan, "Dysfunction of the mitochondrial respiratory chain in the rostral ventrolateral medulla during experimental endotoxemia in the rat," Journal of Biomedical Science, vol. 9, no. 5-6, pp. 542-548, 2002. 
[9] T. Maekawa, Y. Fujii, D. Sadamitsu et al., "Cerebral circulation and metabolism in patients with septic encephalopathy," American Journal of Emergency Medicine, vol. 9, no. 2, pp. 139-143, 1991.

[10] D. Wong and K. Dorovini-Zis, "Expression of vascular cell adhesion molecule-1 (VCAM-1) by human brain microvessel endothelial cells in primary culture," Microvascular Research, vol. 49, no. 3, pp. 325-339, 1995.

[11] I. M. Kafa, I. Ari, and M. A. Kurt, "The peri-microvascular edema in hippocampal CA1 area in a rat model of sepsis," Neuropathology, vol. 27, no. 3, pp. 213-220, 2007.

[12] L. A. Eidelman, D. Putterman, C. Putterman, and C. L. Sprung, "The spectrum of septic encephalopathy: definitions, etioloqies, and mortalities," Journal of the American Medical Association, vol. 275, no. 6, pp. 470-473, 1996.

[13] R. O. Hopkins and J. C. Jackson, "Long-term neurocognitive function after critical illness," Chest, vol. 130, no. 3, pp. 869-878, 2006.

[14] D. N. Nguyen, H. Spapen, F. Su et al., "Elevated serum levels of S-100 $\beta$ protein and neuron-specific enolase are associated with brain injury in patients with severe sepsis and septic shock," Critical Care Medicine, vol. 34, no. 7, pp. 1967-1974, 2006.

[15] O. Piazza, S. Cotena, E. de Robertis, F. Caranci, and R. Tufano, "Sepsis associated encephalopathy studied by MRI and cerebral spinal fluid S100B measurement," Neurochemical Research, vol. 34, no. 7, pp. 1289-1292, 2009.

[16] O. Piazza, E. Russo, S. Cotena, G. Esposito, and R. Tufano, "Elevated S100B levels do not correlate with the severity of encephalopathy during sepsis," British Journal of Anaesthesia, vol. 99, no. 4, pp. 518-521, 2007.

[17] M. van den Boogaard, B. P. Ramakers, N. van Alfen et al., "Endotoxemia-induced inflammation and the effect on the human brain," Critical Care, vol. 14, no. 3, article R81, 2010.

[18] O. Erbaş and D. Taşkıran, "Sepsis-induced changes in behavioral stereotypy in rats, involvement of tumor necrosis factoralpha, oxidative stress, and dopamine turnover," Journal of Surgical Research, vol. I86, no. 1, pp. 262-268, 2014.

[19] J. J. Alexander, A. Jacob, P. Cunningham, L. Hensley, and R. J. Quigg, "TNF is a key mediator of septic encephalopathy acting through its receptor, TNF receptor-1," Neurochemistry International, vol. 52, no. 3, pp. 447-456, 2008.

[20] S. Hofer, C. Bopp, C. Hoerner et al., "Injury of the blood brain barrier and up-regulation of ICAM-1 in polymicrobial sepsis," Journal of Surgical Research, vol. 146, no. 2, pp. 276-281, 2008.

[21] A. J. H. Gearing, I. Hemingway, R. Pigott, J. Hughes, A. J. Rees, and S. J. Cashman, "Soluble forms of vascular adhesion molecules, E-selectin, ICAM-1, and VCAM-1: pathological significance," Annals of the New York Academy of Sciences, vol. 667, pp. 324-331, 1992.

[22] A. J. H. Gearing and W. Newman, "Circulating adhesion molecules in disease," Immunology Today, vol. 14, no. 10, pp. 506-512, 1993.

[23] R. Pigott, L. P. Dillon, I. H. Hemingway, and A. J. H. Gearing, "Soluble forms of E-selectin, ICAM-1 and VCAM-1 are present in the supernatants of cytokine activated cultured endothelial cells," Biochemical and Biophysical Research Communications, vol. 187, no. 2, pp. 584-589, 1992.

[24] H. C. Cowley, D. Heney, A. J. H. Gearing, I. Hemingway, and N. R. Webster, "Increased circulating adhesion molecule concentrations in patients with the systemic inflammatory response syndrome: a prospective cohort study," Critical Care Medicine, vol. 22, no. 4, pp. 651-657, 1994.

[25] R. C. Bone, R. A. Balk, F. B. Cerra et al., "Definitions for sepsis and organ failure and guidelines for the use of innovative therapies in sepsis. The ACCP/SCCM Consensus Conference Committee. American College of Chest Physicians/Society of Critical Care Medicine," Chest, vol. 101, no. 6, pp. 1644-1655, 1992.

[26] M. E. Charlson, P. Pompei, K. A. Ales, and C. R. MacKenzie, "A new method of classifying prognostic comorbidity in longitudinal studies: development and validation," Journal of Chronic Diseases, vol. 40, no. 5, pp. 373-383, 1987.

[27] J.-L. Vincent, R. Moreno, J. Takala et al., “The SOFA (SepsisRelated Organ Failure Assessment) score to describe organ dysfunction/failure," Intensive Care Medicine, vol. 22, no. 7, pp. 707-710, 1996.

[28] W. A. Knaus, E. A. Draper, D. P. Wagner, and J. E. Zimmerman, "APACHE II: a severity of disease classification system," Critical Care Medicine, vol. 13, no. 10, pp. 818-829, 1985.

[29] C.-T. Kung, S.-Y. Hsiao, C.-M. Su et al., "Serum adhesion molecules as predictors of bacteremia in adult severe sepsis patients at the emergency department," Clinica Chimica Acta, vol. 421, pp. 116-120, 2013.

[30] C. L. Sprung, P. N. Peduzzi, C. H. Shatney et al., "Impact of encephalopathy on mortality in the sepsis syndrome," Critical Care Medicine, vol. 18, no. 8, pp. 801-806, 1990.

[31] M. J. Whalen, L. A. Doughty, T. M. Carlos, S. R. Wisniewski, P. M. Kochanek, and J. A. Carcillo, "Intercellular adhesion molecule-1 and vascular cell adhesion molecule-1 are increased in the plasma of children with sepsis-induced multiple organ failure," Critical Care Medicine, vol. 28, no. 7, pp. 2600-2607, 2000 .

[32] M. Guarneri, R. Riccobene, R. Arsena et al., "Flow mediated dilation, endothelial and inflammatory biomarkers in hypertensives with chronic kidney disease," Journal of Hypertension, vol. 28, article el18, 2010.

[33] N. J. Abbott, L. Ronnback, and E. Hansson, "Astrocyteendothelial interactions at the blood-brain barrier," Nature Reviews Neuroscience, vol. 7, no. 1, pp. 41-53, 2006.

[34] B. Jeppsson, H. R. Freund, and Z. Gimmon, "Blood-brain barrier derangement in sepsis: cause of septic encephalopathy?" American Journal of Surgery, vol. 141, no. 1, pp. 136-142, 1981.

[35] M. C. Papadopoulos, F. J. Lamb, R. F. Moss, D. C. Davies, D. Tighe, and E. D. Bennett, "Faecal peritonitis causes oedema and neuronal injury in pig cerebral cortex," Clinical Science, vol. 96, no. 5, pp. 461-466, 1999.

[36] F. A. Bozza, P. Garteiser, M. F. Oliveira et al., "Sepsis-associated encephalopathy: a magnetic resonance imaging and spectroscopy study," Journal of Cerebral Blood Flow and Metabolism, vol. 30, no. 2, pp. 440-448, 2010.

[37] T. Sharshar, R. Carlier, F. Bernard et al., "Brain lesions in septic shock: a magnetic resonance imaging study," Intensive Care Medicine, vol. 33, no. 5, pp. 798-806, 2007.

[38] S. A. Hamed, E. A. Hamed, and M. M. Abdella, "Septic encephalopathy: relationship to serum and cerebrospinal fluid levels of adhesion molecules, lipid peroxides and S-100B protein," Neuropediatrics, vol. 40, no. 2, pp. 66-72, 2009. 
[39] J. Greenwood, S. J. Heasman, J. I. Alvarez, A. Prat, R. Lyck, and B. Engelhardt, "Review: leucocyte-endothelial cell crosstalk at the blood-brain barrier: a prerequisite for successful immune cell entry to the brain," Neuropathology and Applied Neurobiology, vol. 37, no. 1, pp. 24-39, 2011.

[40] K. D. O’Brien, M. D. Allen, T. O. McDonald et al., "Vascular cell adhesion molecule-1 is expressed in human coronary atherosclerotic plaques: implications for the mode of progression of advanced coronary atherosclerosis," Journal of Clinical Investigation, vol. 92, no. 2, pp. 945-951, 1993.

[41] S. Blankenberg, H. J. Rupprecht, C. Bickel et al., "Circulating cell adhesion molecules and death in patients with coronary artery disease," Circulation, vol. 104, no. 12, pp. 1336-1342, 2001. 

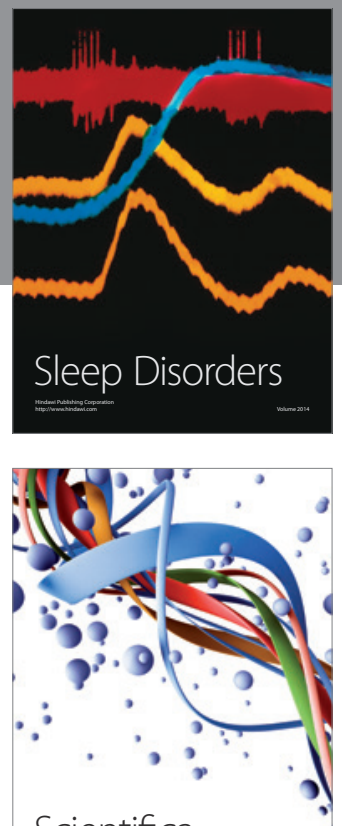

Scientifica
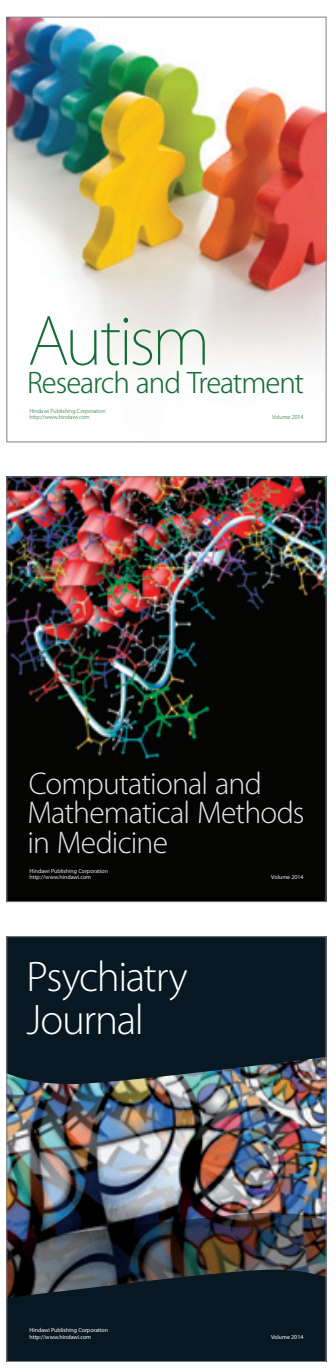
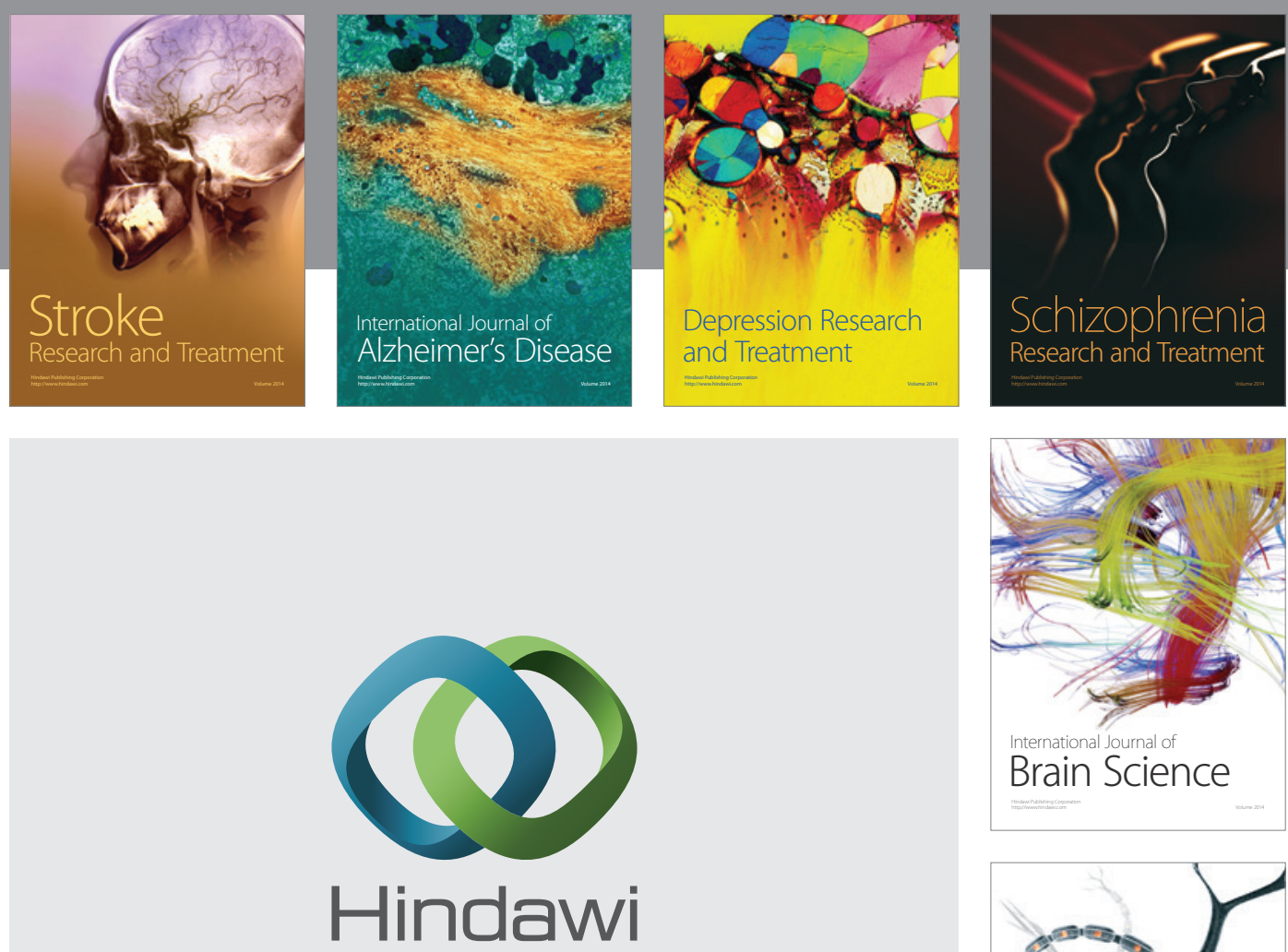

Submit your manuscripts at

http://www.hindawi.com
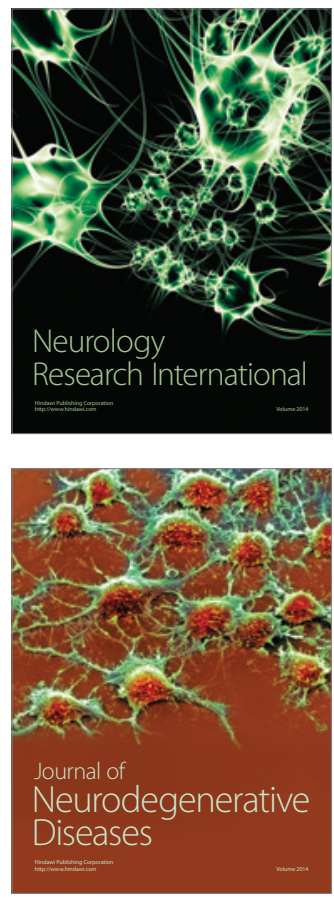

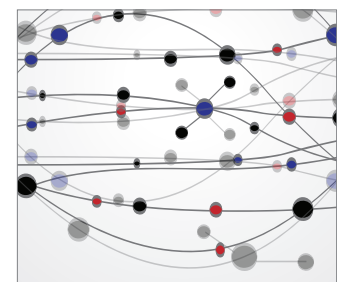

The Scientific World Journal
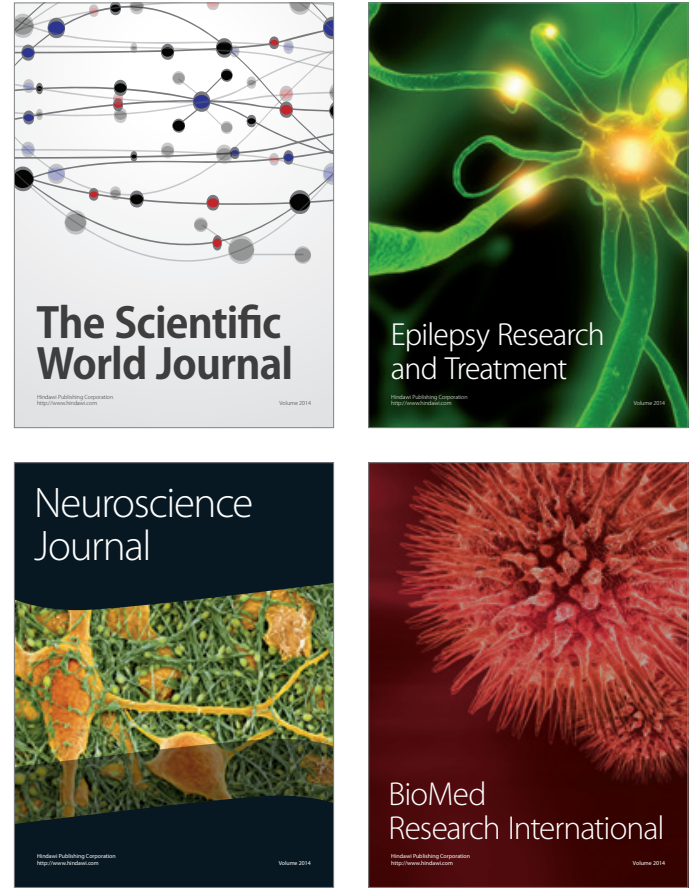

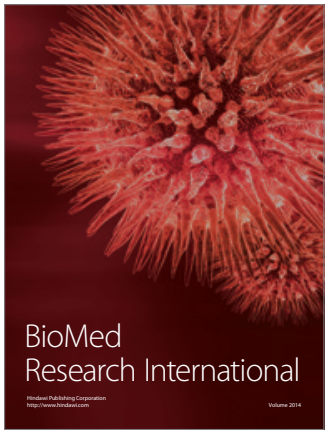

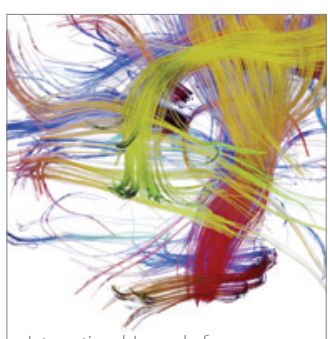

Brain Science

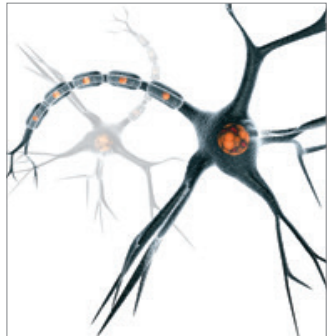

Neural Plasticity
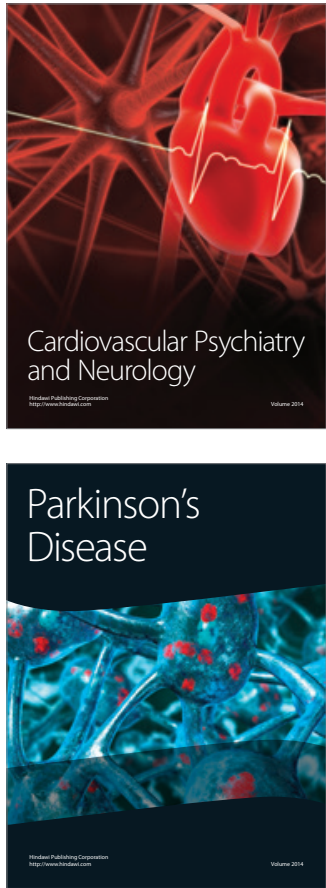